\title{
Vitrification of Human In-Vitro Matured Oocytes: Effects on Mitochondrial Ultrastructure and Oxygen Consumption
}

\author{
Shubiao Han, Wei Han, Xiaodong Zhang, Junxia Liu, Guoning Huang \\ Chongqing Health Center for Women and Children, Chongqing 400010, China
}

\begin{abstract}
Background: This study was conducted to evaluate the impact of vitrification on mitochondrial of human IVM oocytes.

Methods: A total of 401 immature oocytes were obtained from ovarian stimulated cycles, which were randomly divided into fresh and vitrification groups after IVM. According to the cultured time after thawing, the vitrification groups were divided into 0 hours $(0 \mathrm{~h}), 2$ hours $(2 \mathrm{~h})$, or 4 hours $(4 \mathrm{~h})$ subgroups. Mitochondrial morphology and oxygen consumption were compared among the four groups. After fertilization by ICSI, normal fertilization, cleaved embryos, and blastocyst formation rate were also calculated.

Results: The mean gray value of mitochondria structure was significantly decreased in $0 \mathrm{~h}$ and $2 \mathrm{~h}$ groups when compared to control group $(0.48 \pm 0.09,0.50 \pm 0.36$ vs. $0.61 \pm 0.12$, respectively; $\mathrm{P}<0.05)$, and recovered $(0.61 \pm 0.24$ vs. $0.61 \pm 0.12, \mathrm{P}>0.05)$ in $4 \mathrm{~h}$ group. In addition, oxygen consumption was also significantly decreased in $0 \mathrm{~h}$ and $2 \mathrm{~h}$ groups compared to fresh $(2.91 \pm 0.77 \mathrm{fmol} / \mathrm{s}$, $3.26 \pm 1.34 \mathrm{fmol} / \mathrm{s}$ vs. $3.96 \pm 1.44 \mathrm{fmol} / \mathrm{s}$, respectively; $\mathrm{P}<0.05)$, and recovered after $4 \mathrm{~h}$ culture $(3.96 \pm 1.44 \mathrm{fmol} / \mathrm{s}$ vs. $4.41 \pm 1.38$ $\mathrm{fmol} / \mathrm{s}$, respectively; P > 0.05). The percentage of normal fertilization and cleaved embryos were no differences among the four groups, however, blastocyst development rate was significantly lower in $0 \mathrm{~h}$ group.
\end{abstract}

Conclusion: These results indicate that during the vitrification process, the oxygen consumption and mitochondrial structure of oocytes may undergo temporary dynamic changes, but appear to recover by 4 hours.

Keywords: Vitrification; Metaphase II Oocytes; Oxygen Consumption; Mitochondrial Structure; In-Vitro Maturation.

\section{INTRODUCTION}

Since the first human birth following slow freezing of oocytes occurred in 1986 (Chen, 1986), the categorization of oocyte cryopreservation has changed from "experimental" to being endorsed in 2012 by the American Society for Reproductive Medicine (ASRM) and the European Society of Human Reproduction and Embryology (ESHRE), for clinical use. The success rate of oocyte cryopreservation is still far from satisfactory (Hosseini and NasrEsfahani, 2016). Although warmed oocytes possess a fertilization rate similar to that of fresh non-donor oocytes and a survival rate similar to that of embryo cryopreservation (Borini et al., 2006; Porce, 2001; Selman et al., 2006), the cleavage rates after fertilization and clinical outcomes after ET have been shown to be lower than those fresh non-donor oocytes (Azambuja et al., 2011; Cobo and Diaz, 2011; Trokoudes et al., 2011). The potential injury may have occurred during the vitrification-warming procedure, which may undermine oocyte viability via cytoskeletal changes, cryoprotectant osmotic stress and toxicity, meiotic spindle damage and unbalanced calcium homeostasis. Recent research by Nohales-Corcoles et al. (2016) found that the alteration of the redox potential (measured by the $\mathrm{FAD} / \mathrm{NAD}(\mathrm{P}) \mathrm{H}$ ratio) of the higher oxidative stage in oocytes after vitrification procedures, could eventually impair blastocyst development. However, another reason commonly overlooked is inappropriate timing of insemination after thawing.

Oocytes have been proven to be more difficult to vitrify and warm than embryos, as they are large cells with a low surface areato-volume ratio and therefore a high propensity for intracellular ice formation (Paynter et al., 1999); additionally, oocytes are poorly permeable to CPA and water, and have a lower resistance to freezing injury than do zygotes (Ghetler et al., 2005). Their internal structures are also extremely susceptible to cryo-damage. Hosseini et al. (2015) used a mouse oocyte model and found that cytoplasmic insufficiency was the primary cause of poor competence of matured oocytes after vitrification.

Mitochondria are specialized organelles in the oocyte cytoplasm that catalyze the formation of adenosine trisphosphate (ATP), which provides almost all of the energy for fertilization and embryonic development; and they thereby constitute a sensitive index of cellular damage because they are easily influenced by their environment (Gualtieri et al., 2009). Our previous study showed

This is an Open Access article published by World Scientific Publishing Company. It is distributed under the terms of the Creative Commons AttributionNonCommercial-NoDerivatives 4.0 (CC BY-NC-ND) License which permits use, distribution and reproduction, provided that the original work is properly cited, the use is non-commercial and no modifications or adaptations are made.

Received 21 January 2019; Accepted 30 July 2019; Published 20 August 2019

${ }^{*}$ Correspondence should be addressed to: Dr. Guoning Huang, Chongqing Health Center for Women and Children, 64 Jintangjie, Yuzhong, Chongqing 400010, China. Email: gnhuang217@sina.com 
that the mitochondrial membrane potential of human unfertilized MII oocytes was decreased after vitrification, and that recovery occurred spontaneously in culture 4 hours after thawing (Chen et al., 2012). These results showed that oocytes may need some time to repair mitochondrial damage during vitrification. Assessment by transmission electron microscopy (TEM), therefore, appears to be effective in estimating how vitrification damages oocyte mitochondrial structure during freeze-thawing. Shiku et al. (2001) succeeded in non-invasively determining oxygen consumption by individual oocytes and embryos using a scanning electrochemical microscopy (SECM) measuring system that enables the evaluation of mitochondrial activity in oocytes and embryos.

The objectives of the present study were to assess the impact of vitrification on mitochondrial ultrastructure and oxygen consumption in human vitrified MII oocytes compared to nonvitrified (fresh) oocytes, and to access times after thawing. The optimal time for insemination after vitrification.

\section{MATERIALS AND METHODS}

\section{Human oocyte collection}

The immature unfertilized human oocytes in this study were donated by women receiving ART in our center between January 2014 and December 2014. Donated oocytes from women aged between 20 and 35 underwent ICSI because of a simple male factor. Only immature oocytes at the time of the ICSI which were not used for in vitro fertilization were used in this study. The sperms were obtained from residual sperms of donor cycles on the same day that ICSI was performed, and the partners' sperms used for the original insemination were the same sperms as used in the subsequent insemination with thawed IVM oocytes. All procedures were performed according to the Helsinki Declaration on Human Experimentation. The study was approved by the Commission for Medical Ethics of the Chongqing Obstetrics and Gynecology Hospital (approval reference number RGI-ZA201404).

\section{Oocyte randomization and group assignments}

A total of 461 metaphase I (MI) oocytes were collected and cultured for $20-24$ hours at $37{ }^{\circ} \mathrm{C}$ in an atmosphere of $5 \% \mathrm{CO}_{2}$ and $20 \% \mathrm{O}_{2}$ with high humidity. After culture the presence of first polar body was assessed under the stereo microscope and then used for further study.
Each oocyte that underwent in-vitro maturation (IVM) was assigned an order number in the study group. A randomization sequence was created using Stata 12.0 (StataCorp, College Station, TX) software.

A total of $401 \mathrm{MI}$ oocytes were collected from 311 patients: 286 oocytes matured after IVM and were randomly divided into four groups, 67 oocytes in control group, 71 oocytes in $0 \mathrm{~h}$ group, 74 oocytes in $2 \mathrm{~h}$ group, and 76 oocytes in $4 \mathrm{~h}$ group. The oocytes in 0,2 and $4 \mathrm{~h}$ groups were vitrified. Thirty-six oocytes for the measurement of oxygen consumption were arranged at 0,2 and $4 \mathrm{~h}$ after warming. A flowchart of the trial procedure is depicted in Fig. 1.

\section{Vitrification/warming of human oocytes}

The Kitazato Vitrification Kit (Kita, Toyota, Japan) was used for oocyte vitrification, and method was modified based on Kitazato's protocol. Oocytes were equilibrated in VS 1 solution for $5 \mathrm{~min}$ at room temperature before exposure to the vitrification solution (VS 2). After equilibration, oocytes were immersed in VS 2 solution for $30 \mathrm{sec}$. The oocytes were then placed on a Cryotop sheet and excess vitrification solution was removed by aspiration with a pipette before the carrier was immersed in $\mathrm{LN}_{2}$.

The Kitazato Thaw Kit (Kita, Toyota, Japan) was used for oocyte warming. The oocytes warming method was modified based on Kitazato's protocol. Oocytes were merged into a dish containing $3 \mathrm{ml}$ of $1.0 \mathrm{~mol} / \mathrm{L}$ sucrose at $37^{\circ} \mathrm{C}$ for $1 \mathrm{~min}$. Then transferred sequentially to 0.5 and $0.25 \mathrm{~mol} / \mathrm{L}$ sucrose solutions at room temperature for 3 min each. The oocytes were washed several times in Quinn's 1024 (Cooper Surgical, CT, USA) solution, and placed in G1 medium (Vitrolife, Kungsbacka, Sweden) for further experimentation. Oocytes that appeared rounded, with a homogeneous cytoplasm, and that possessed an intact oolemma and a continuous zona pellucida (ZP) were recognized as oocytes that survived thawing.

\section{Transmission electron microscopy}

Oocytes from each group were fixed and processed for TEM as described by Shahedi et al. (2013) Oocyte fixation was performed in $1.5 \%$ glutaraldehyde (Sigma, St. Louis, USA) in a phosphate-buffered saline (PBS) solution. After fixation for $2-5$ days at $4{ }^{\circ} \mathrm{C}$, the samples were rinsed in PBS, post-fixed with $1 \%$ osmium tetroxide (SIC) in PBS and rinsed again in PBS. Oocytes were then embedded in small blocks of $2 \%$ agar (Sigma, St. Louis, USA) of about $5 \times 5 \times 1 \mathrm{~mm}$ in size,

Fig. 1. Flowchart of the trial procedure: 36 oocytes from 0,2 and $4 \mathrm{~h}$ groups were selected for measurement of oxygen consumption at 0,2 and $4 \mathrm{~h}$ after warming, as this method is non-invasive.

Notes: IVM = in vitro maturation, TEM = transmission electron microscopy, ICSI = intracytoplasmic sperm injection, SECM = scanning electrochemical microscopy.

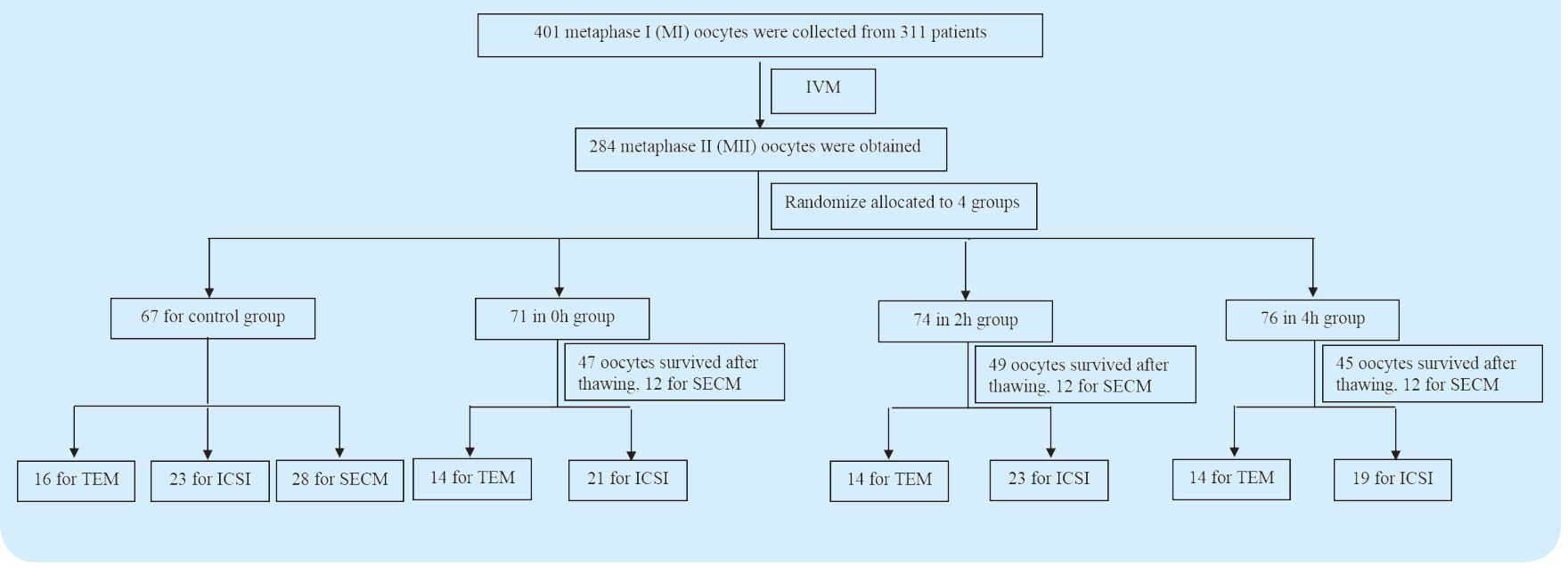


dehydrated in an ascending series of ethanol, immersed in propylene oxide for solvent substitution, and embedded in Epon 812 (SIC); ultrathin sections (60-90 $\mathrm{nm}$ ) were cut with a diamond knife, mounted on copper grids and contrasted with saturated uranyl acetate followed by lead citrate. The sections were examined and photographed using a transmission electron microscope (Hitachi- 600, Japan). Five pictures were randomly selected from TEM and used to calculate the gray value of every mitochondria in each group, and 6 mitochondria were analyzed by random selection from each picture, then the gray value of each mitochondria calculated by Image J software (Softonic, Spain), follow the instruction of software (Schneider et al., 2012).

\section{Oocyte oxygen consumption}

Before the measurement of oocyte oxygen consumption, preparation was as follows: oocyte was initially transferred to a plate with coneshaped microwells, with Quinn's 1024 (Cooper Surgical, CT, USA) $+10 \%$ HSA (Vitrolife, Kungsbacka, Sweden) medium. A microdisk electrode was used to scan in the Z-direction from the bottom of a microwell. The motor-driven XYZ-stage was located on the microscope stage for electrode tip scanning, and the XYZ stage and potentiostat were controlled by computer. The oxygen consumption rate of oocytes was then calculated with software, using an algorithm based upon the spherical diffusion theory. The measurement of oxygen consumption for each oocyte took approximately $40 \mathrm{sec}$. Overall; it took less than $3 \mathrm{~min}$ to perform three measurements, which were then used to calculate the average respiratory activity of each oocyte.

\section{Oocyte fertilization and embryo culture}

The oocytes from each group were inseminated by ICSI, fertilization was examined 16-18 h after ICSI and zygotes were cultured in G1 medium (Vitrolife, Kungsbacka, Sweden) supplemented with $10 \%$ $\mathrm{HSA}$ at $37{ }^{\circ} \mathrm{C}$ in a humidified atmosphere of $5 \% \mathrm{CO}_{2}$ and $5 \% \mathrm{O}_{2}$ until the 6 days after inseminations.

\section{Statistical analyses}

All results were analyzed using Stata12.0 statistical software (StataCorp, College Station, TX). Analyses of oxygen consumption were performed by repeated measurements. $\mathrm{P}<0.05$ was considered to be statistically significant.

\section{RESULTS}

A total of $401 \mathrm{MI}$ oocytes were collected from 311 patients (mean patient age $31.04 \pm 3.48$ ): $71.32 \%$ of MI oocytes (286/401) matured after IVM, 221MII oocytes were frozen, and $63.80 \%$ of MII oocytes $(141 / 221)$ survived after thawing.
Table 1. Oxygen consumption rates of human IVM oocytes at various times after warming.

\begin{tabular}{lcc}
\hline Group & No. of oocytes measured & Oxygen consumption rate $\left(\mathbf{F} \times \mathbf{1 0} \mathbf{1 5}^{15} \mathbf{\mathbf { m o l ~ S } ^ { - 1 } )}\right.$ \\
\hline $0 \mathrm{~h}$ & 36 & $2.91 \pm 0.77^{*}$ \\
$2 \mathrm{~h}$ & 36 & $3.26 \pm 1.34^{*}$ \\
$4 \mathrm{~h}$ & 36 & $3.96 \pm 1.44^{*}$ \\
$\mathrm{C}$ & 28 & $4.41 \pm 1.38^{*}$ \\
\hline $\mathrm{P}<0.05$ between and $^{*}$ & & \\
& &
\end{tabular}

\section{The vitrification-warming causes the temporary decrease of oocyte oxygen consumption}

The oocyte oxygen consumption rate was significantly decreased in the 0 and $2 \mathrm{~h}$ groups when compared to the control group $(2.91 \pm$ $0.77 \mathrm{fmol} / \mathrm{s}, 3.26 \pm 1.34 \mathrm{fmol} / \mathrm{s}$ vs. $3.96 \pm 1.44 \mathrm{fmol} / \mathrm{s}$, respectively; $\mathrm{P}<0.05)$, there was no significant difference between the control group and the $4 \mathrm{~h}$ group $(3.96 \pm 1.44 \mathrm{fmol} / \mathrm{s}$ vs. $4.41 \pm 1.38 \mathrm{fmol} / \mathrm{s}$, $\mathrm{P}>0.05$ ) (Table 1). These results show that vitrification-warming causes a temporary decrease of oocyte oxygen consumption, but this recovers after $4 \mathrm{~h}$ of culture.

\section{The vitrification-warming causes the temporary mitochondrial ultrastructure abnormity}

The oocyte oxygen consumption is associated with the mitochondrial function, therefore the mitochondrial ultrastructure was analyzed by the TEM. In the TEM photograph (Fig. 1), we observed tiny gaps (white line or amorphous shape) immediately after warming and 2 hours after culture; these disappeared after 4 hours of in-vitro culture. The dynamic photos were consistent with the results from the oxygen consumption rate. To quantify the changes of mitochondrial tiny gaps, an image analysis software was used, this also shows the same trend as obtained by TEM photos analysis.

Six mitochondria were randomly selected for five pictures in each group, and were analyzed by random selection from each picture, and a total of 30 mitochondrial were calculated by Image J software in each group. The gray value was used to quantified the changes of the tiny gaps (Fig. 2), the mean gray value was significantly decreased in the $0 \mathrm{~h}$ and $2 \mathrm{~h}$ groups when compared to the control group $(0.48 \pm 0.09,0.50 \pm 0.36$ vs. $0.61 \pm 0.12$, respectively; $\mathrm{P}<0.05)$. After 4 hours of culture, the mean gray value recovered to the level without vitrification-warming $(0.61 \pm 0.24$ vs. $0.61 \pm 0.12, \mathrm{P}>0.05)$

Fig. 2. Tiny gaps of human IVM oocytes at various times after warming. (A) Fresh mitochondrial (control group) without tiny gaps. (B) Mitochondrial at $0 \mathrm{~h}$ after oocytes warming with large white lines or amorphous shapes (tiny gaps are denoted by red arrow). (C) Mitochondrial at $2 \mathrm{~h}$ after oocytes warming showing moderate white dots. (D) Mitochondrial at $4 \mathrm{~h}$ after oocytes warming with smaller and fewer tiny gaps. Red circle and green arrow showed the example of gray area for quantified the changes of the tiny gaps.
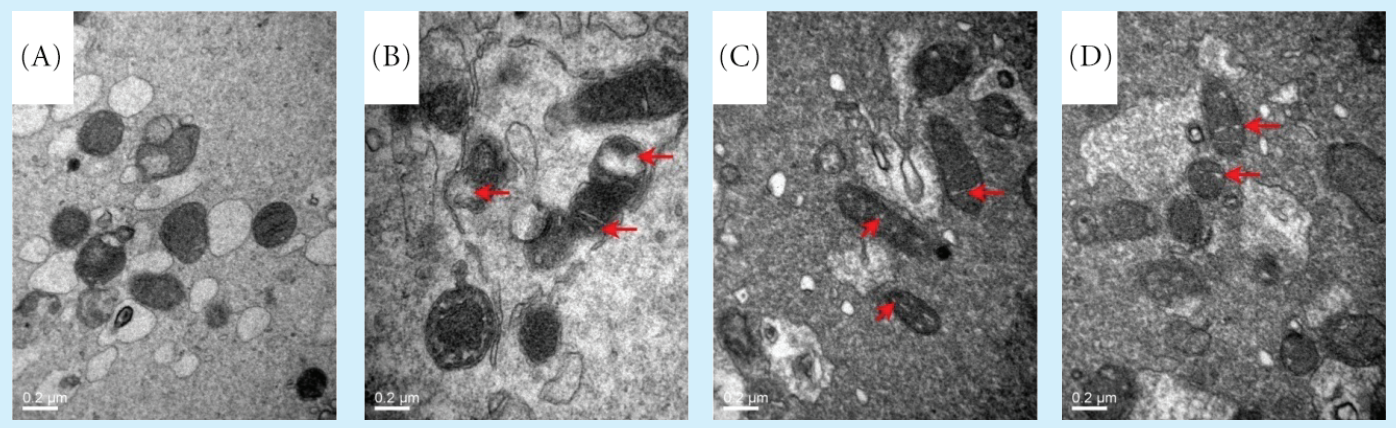
Table 2. Mean gray value of mitochondrial at various times after warming.

\begin{tabular}{lcc}
\hline Group & No. of mitochondrial measured & Mean of gray value \\
\hline $0 \mathrm{~h}$ & 30 & $0.48 \pm 0.09^{*}$ \\
$2 \mathrm{~h}$ & 30 & $0.50 \pm 0.36^{*}$ \\
$4 \mathrm{~h}$ & 30 & $0.61 \pm 0.24^{*}$ \\
Control & 30 & $0.61 \pm 0.12^{*}$ \\
\hline $\mathrm{P}<0.05$ between \# and ${ }^{*}$. &
\end{tabular}

$<0.05$ between $\#$ and ${ }^{*}$

Table 3. Embryo development of human warming oocytes after ICSI.

\begin{tabular}{lcccc}
\hline Group & No. of oocytes & No. fertilized & No. cleaved & No. of blastocysts \\
\hline $0 \mathrm{~h}$ & 21 & $57 \%(12 / 21)$ & $58 \%(7 / 12)$ & $14 \%(1 / 7)$ \\
$2 \mathrm{~h}$ & 23 & $61 \%(14 / 23)$ & $57 \%(8 / 14)$ & $50 \%(4 / 8)$ \\
$4 \mathrm{~h}$ & 19 & $63 \%(12 / 19)$ & $75 \%(9 / 12)$ & $22 \%(2 / 9)$ \\
Control & 23 & $65 \%(15 / 23)$ & $80 \%(12 / 15)$ & $50 \%(6 / 12)$ \\
\hline
\end{tabular}

(Table 2). These results demonstrate that the vitrification-warming causes the temporary mitochondrial ultrastructure inter membrane space abnormity, which was consistent with the oxygen consumption rate.

\section{The effect of vitrification-warming on fertilization and embryo development}

As shown in Table 3, the normal fertilization and cleaved rates show no differences among the four groups.

\section{DISCUSSION}

The primary function of mitochondria is to generate ATP, which is necessary for normal physiological functions, including motility, maintenance of cellular homeostasis, and regulation of cell survival. Many factors have been proven to affect the mitochondria of human oocytes, such as maternal age and vitrification damage (Wilding et al., 2005). Vitrification has been reported to compromise mitochondrial function and reduce ATP content in human and bovine oocytes, which might contribute to poor oocyte development after cryopreservation (Moussa et al., 2014). Our previous study showed that the mitochondrial membrane potential of human unfertilized MII oocytes was decreased after vitrification, and that recovery occurred spontaneously in culture 4 hours after thawing (Chen et al., 2012). The present study was to assess the impact of vitrification on mitochondrial ultrastructure and oxygen consumption in human MII oocytes, and to reveal the function and ultrastructure of mitochondrial changes. The results suggest that 4 hours are needed for oxygen consumption and mitochondrial ultrastructure of oocytes returned to normal.

Importantly, oxygen consumption rate has recently been considered as a metabolic indicator, which reflects the activity of mitochondrial oxidative phosphorylation (OXPHOS) that synthesizes adenosine triphosphate (ATP) (Kurosawa et al., 2016). Although staining with the $\Delta \Psi \mathrm{m}$-specific probe JC-1 detected instantaneous activation or mitochondrial excitability, oxygen consumption as detected by SECM has often been regarded as a better indicator of overall metabolic activity (Tejera et al., 2011).
It has also been demonstrated that oxygen consumption is related to oocyte fertilization capability, which is consistent with previous data regarding higher ATP turnover in oocytes (Ruvulo et al., 2013). Yamanaka et al. (2011) found that the oxygen consumption rate of blastocysts increased with time after warming, and was not observed at $0 \mathrm{~h}$ after warming, but was observed at $24 \mathrm{~h}$, suggesting that mitochondrial functions are restrained or obstructed somewhere during the cryopreservation process. Yoshida et al. (2013) found that when the oxygen consumption rate increases in a group of thawing embryos, developmental rate for blastocyst and freezing ability for cryopreservation will be increased. In this study, comparison of the oxygen consumption rate of human MII oocytes from $0 \mathrm{~h}$ to $4 \mathrm{~h}$ after warming (based on our preliminary experiments and published results) (Chen et al., 2012) found that the oxygen consumption rate was increased with the time lapse, and in $4 \mathrm{~h}$ after warming the oxygen consumption rate is almost the level of the control group. This may be due to the vitrification decreasing mitochondrial potential in human oocytes (Nohales-Corcoles et al., 2016). In addition, the method for oxygen consumption rate measurement is by measuring the oxygen concentration gradient around the oocyte, so this was not affected by oxygen concentration in the culture medium (Ottosen et al., 2007).

The mitochondrial structure is composed of the outer membrane, the inner membrane and the inter membrane space. Outward proton pumping across the inner mitochondrial membrane creates a proton gradient that has two components, a $\Delta \Psi \mathrm{m}$ and a $\mathrm{pH}$ gradient, and the energy stored in either component drives the conversion of ADP to ATP by respiratory chain enzymes (van Blerkom et al., 2002). Human IVM oocytes after vitrification frequently show a typical and immature mitochondrial smooth endoplasmic reticulum aggregates (Liu et al., 2017), and mitochondrial damage (e.g., modifications in shape and surface, swelling, reduced density or alterations in cristae) has been frequently detected in some other mammals subjected to different vitrification protocols (Hyttel et al., 2000; Morato et al., 2008; Wu et al., 2006). In the present study, the inter membrane space was huge and tiny gaps appeared after warm culture, and these may be categorized into alterations of mitochondrial cristae. The gray value was used to quantify the changes in the tiny gaps (Fig. 2), an image analysis software was used for quantification analysis, the same trend was obtained by TEM photoanalysis. Additionally, the changes in organellar microtopography are not only affected by the methods of cryopreservation used (Khalili et al., 2012), but also by some of the specific details of methodologies involved in cryopreservation (Davidson et al., 2014).

The results demonstrated optimal ICSI time post warming. After 2 hours' recovery, the cleavage rate may recover to normal, but after 4 hours of post warming, the blastocysts formation rate was reduced, maybe due to the longer waiting time for ICSI leading to the aging of oocytes. Also, due to the small sample size of the present study, there is a need for further studies. Our recent unpublished results confirm that after 5 hours of post warming culture, mitochondrial aggregation was found in oocyte cytoplasm. For further research, pregnancy and live birth rates would be set as the primary endpoint for comparing the effect of optimal times for post warming culture.

The limitation of these studies is that the human oocytes which were obtained were in vitro- and failed-matured. Hence, it is possible that such oocytes may not represent healthy human oocytes. In addition, it has been reported that cryopreservation significantly increases ROS production in young mouse oocytes but not in oocytes from reproductively older mice (Nohales-Corcoles et al., 2016), and that may limit the use of oxygen consumption in all patients. Due to the small sample size of the present study, these results can only be regarded as preliminary. 
In conclusion, the vitrification-warming process can cause the temporary oxygen consumption and mitochondrial ultrastructure abnormalities, but they can recover after 4 hours of culture.

\section{ACKNOWLEDGEMENTS}

We thank Wang Hong of Chongqing Medical University for transmission electron microscopic analysis.

\section{AUTHORS' ROLES}

Han Shubiao and Huang Guoning designed the study. Zhang Xiaodong analyzed the data. Han Shubiao, Han Wei, Zhang Xiaodong, Liu Junxia, and Liu Weiwei participated in the experiment. Han Shubiao, Han Wei and Zhang Xiaodong wrote the paper.

\section{FUNDING}

This study was supported by the basic and prospective Foundation of the Chongqing province, China (cstc2016jcy JAX0007), Foundation of the Chinese Medical Association of Clinical Medicine Research, China (No. 16020350651), and the Basic and Prospective Foundation of the Yuzhong District, Chongqing province, China (No. 20150141).

\section{CONFLICT OF INTEREST}

The authors declare that there is no conflict of interest.

\section{REFERENCES}

Azambuja R, et al. Experience of freezing human oocytes using sodium-depleted media. Reprod Biomed Online. 2011;22(1): 83-7.

Borini A, et al. Cumulative pregnancy rates resulting from the use of fresh and frozen oocytes: 7 years' experience. Reprod Biomed Online. 2006;12(4):481-6.

Chen C. Pregnancy after human oocyte cryopreservation. Lancet. 1986;1(8486):884-6.

Chen $\mathrm{C}$, et al. Effect of vitrification on mitochondrial membrane potential in human metaphase II oocytes. J Assist Reprod Genet. 2012;29(10):1045-50.

Cobo A, Diaz C. Clinical application of oocyte vitrification: a systematic review and meta-analysis of randomized controlled trials. Fertil Steril. 2011;96(2):277-85.

Davidson AF, Benson JD, Higgins AZ. Mathematically optimized cryoprotectant equilibration procedures for cryopreservation of human oocytes. Theor Biol Med Model. 2014;11:13.

Ghetler Y, et al. The effect of chilling on membrane lipid phase transition in human oocytes and zygotes. Hum Reprod. 2005;20(12):3385-9.

Gualtieri R, et al. Slow cooling of human oocytes: ultrastructural injuries and apoptotic status. Fertil Steril. 2009;91(4):1023-34.

Hosseini SM, Nasr-Esfahani MH. What does the cryopreserved oocyte look like? A fresh look at the characteristic oocyte features following cryopreservation. Reprod Biomed Online. 2016;32(4):377-87.

Hosseini SM, et al. Cytoplasmic, rather than nuclear-DNA, insufficiencies as the major cause of poor competence of vitrified oocytes. Reprod Biomed Online. 2015;30(5):549-52.

Hyttel P, Vajta G, Callesen H. Vitrification of bovine oocytes with the open pulled straw method: ultrastructural consequences. Mol Reprod Dev. 2000;56(1):80-8.
Khalili MA, et al. Ultrastructure of human mature oocytes after vitrification. Eur J Histochem. 2012;56(3):e38.

Kurosawa $\mathrm{H}$, et al. Development of a new clinically applicable device for embryo evaluation which measures embryo oxygen consumption. Hum Reprod. 2016;31(10):2321-30.

Liu $\mathrm{MH}$, et al. Ultrastructural changes and methylation of human oocytes vitrified at the germinal vesicle stage and matured in vitro after thawing. Gynecol Obstet Invest. 2017;82(3):252-61.

Morato R, Mogas T, Maddox-Hyttel P. Ultrastructure of bovine oocytes exposed to Taxol prior to OPS vitrification. Mol Reprod Dev. 2008;75(8):1318-26.

Moussa M, et al. Cryopreservation of mammalian oocytes and embryos: current problems and future perspectives. Sci China Life Sci. 2014;57(9):903-14.

Nohales-Corcoles $\mathrm{M}$, et al. Impact of vitrification on the mitochondrial activity and redox homeostasis of human oocyte. Hum Reprod. 2016;31(8):1850-8.

Ottosen LD, et al. Murine pre-embryo oxygen consumption and developmental competence. J Assist Reprod Genet. 2007;24(8): 359-65.

Paynter SJ, et al. Permeability characteristics of human oocytes in the presence of the cryoprotectant dimethylsulphoxide. Hum Reprod. 1999;14(9):2338-42.

Porcu E. Oocyte freezing. Semin Reprod Med. 2001;19(3):221-30.

Ruvolo G, et al. New molecular markers for the evaluation of gamete quality. J Assist Reprod Genet. 2013;30(2):207-12.

Schneider CA, Rasband WS, Eliceiri KW. NIH Image to ImageJ: 25 years of image analysis. Nat Methods. 2012;9(7):671-5.

Selman $\mathrm{H}$, et al. Ongoing pregnancies after vitrification of human oocytes using a combined solution of ethylene glycol and dimethyl sulfoxide. Fertil Steril. 2006;86(4):997-1000.

Shahedi A, et al. Ultrastructure of in vitro matured human oocytes. Iran Red Crescent Med J. 2013;15(12):e7379.

Shiku H, et al. Oxygen consumption of single bovine embryos probed by scanning electrochemical microscopy. Anal Chem. 2001; 73(15):3751-8.

Tejera A, et al. Oxygen consumption is a quality marker for human oocyte competence conditioned by ovarian stimulation regimens. Fertil Steril. 2011;96(3):618-623 e2.

Trokoudes KM, Pavlides C, Zhang X. Comparison outcome of fresh and vitrified donor oocytes in an egg-sharing donation program. Fertil Steril. 2011;95(6):1996-2000.

Van Blerkom J, et al. Domains of high-polarized and low-polarized mitochondria may occur in mouse and human oocytes and early embryos. Hum Reprod. 2002;17(2):393-406.

Wilding M, Di Matteo L, Dale B. The maternal age effect: a hypothesis based on oxidative phosphorylation. Zygote. 2005;13(4):317-23.

$\mathrm{Wu} \mathrm{C}$, et al. Effects of cryopreservation on the developmental competence, ultrastructure and cytoskeletal structure of porcine oocytes. Mol Reprod Dev. 2006;73(11):1454-62.

Yamanaka M, et al. Developmental assessment of human vitrifiedwarmed blastocysts based on oxygen consumption. Hum Reprod. 2011;26(12):3366-71.

Yoshida H, Abe H, Arima T. Quality evaluation of IVM embryo and imprinting genes of IVM babies. J Assist Reprod Genet. 2013; 30(2):221-5. 